\title{
Sulla diffusione areale di un fenomeno di variazione morfo-fonetica nei dialetti campani
}

\author{
Maturi, Pietro ; Schmid, Stephan
}

Posted at the Zurich Open Repository and Archive, University of Zurich ZORA URL: https://doi.org/10.5167/uzh-117696

Book Section

Originally published at:

Maturi, Pietro; Schmid, Stephan (2003). Sulla diffusione areale di un fenomeno di variazione morfofonetica nei dialetti campani. In: Sánchez Miret, Fernando. Actas XXIII del Congreso Internacional de Lingüística y Filología Románica. Tübingen: Niemeyer, 221-233. 


\title{
Pietro Maturi / Stephan Schmid
}

\section{Sulla diffusione areale di un fenomeno di variazione morfo-fonetica nei dialetti campani}

\author{
1 Introduzione
}

Con questo contributo intendiamo presentare nuovi dati empirici su un fenomeno di variazione di altezza vocalica che abbiamo riscontrato in alcuni dialetti della Campania settentrionale e che abbiamo descritto in lavori recenti (Maturi / Schmid 1999; Maturi / Schmid 2001). Tale fenomeno di variazione si presenta come un caso tipico di polimorfia (cfr. Radtke 1997: 32-33), intesa come variazione non definita a priori in base a parametri sociolinguistici: infatti, nel nostro caso pare che la stessa alternanza tra gli allomorfi di un numero limitato di parole funzionali si manifesti non tanto nella dimensione diatopica (dove distinguerebbe le parlate di varie località della stessa area), quanto nella variazione interna alle varietà basilettali dei dialetti locali, se non addirittura a livello intraindividuale, cioè nella produzione linguistica di uno stesso parlante.

Gli obiettivi della nostra ricerca sono essenzialmente due. Da un lato si vuole descrivere nel modo più esatto possibile il fenomeno sotto esame, osservando la sua variazione ai vari livelli della dimensione diatopica (regione Campania, province e singole località), nonché a livello intraindividuale. Dall'altro lato ci prefiggiamo di interrogarci sui meccanismi sottostanti a questo particolare tipo di allomorfia, sottoponendo alla verifica strumentale dei dati l'ipotesi di un suo condizionamento fonetico-prosodico.

Il lavoro è articolato nel seguente modo: in un primo momento illustreremo la polimorfia dai punti di vista descrittivo e variazionale, basandoci sulla documentazione dialettologica tradizionale nonché su alcuni contributi recenti (§2); successivamente presenteremo i dati di una ricerca empirica che si avvale dell'apporto della fonetica acustica ( $\$ 3)$, per discutere infine la natura della polimorfia alla luce dei nostri risultati $(\S 4)$.

\section{Polimorfia di alcune parole funzionali nei dialetti campani}

2.1 La variazione di altezza vocalica

La polimorfia qui indagata si presenta come variazione timbrica nelle vocali di alcune parole funzionali, per lo più clitici monosillabici; tali vocali, che siano anteriori o posteriori, oscillano grosso modo tra il grado di apertura medio e quello alto.

Nel caso delle vocali posteriori, l'oscillazione tra $[\mathrm{o}]$ e $[\mathrm{u}]$ caratterizza le seguenti classi di parole funzionali monosillabiche: l'articolo determinativo singolare maschile (1) e neutro (2), il pronome clitico di complemento diretto singolare maschile (3) e neutro (4), 
l'articolo indeterminativo maschile (5) e la negazione «non» (6), come appare nelle seguenti forme dal dialetto di Sant'Agata de' Goti (BN).
(1) [o 'so:lə]
(2) [o 'sa:lo]
var. [u 'so:le]
«il sole»
(3) [o 've:kə]
var. [u 's:ailə]
«il sale»
(4) [o 'b:e:kə]
var. [u 've:kə]
«lo vedo (m.)»
(5) [no wa'ko:nə]
var. [u 'bie:kə]
«lo vedo (n.)»
(6) [no 's:at: $\left.\int ə\right]$
var. [nu wa' Ko:nə]
«un ragazzo»
var. [nu 'srat: $\left.\int ə\right]$
«non so»

Dal punto di vista della descrizione grammaticale va precisato che nei dialetti campani esiste per le categorie (1), (2), (3) e (4) un ulteriore allomorfo, determinato in modo univoco dal contesto fonotattico. Infatti, articoli e pronomi hanno la forma citata soltanto se la parola successiva inizia con una consonante; nel contesto prevocalico viene automaticamente selezionato l'allomorfo con la consonante etimologica, in forma apocopata e di lunghezza variabile (p. es. l'uosso o ll'uosso «l'osso»). Il genere neutro (2, 4) è riservato a referenti con la proprietà semantica di nomi massa e viene segnalato mediante il raddoppiamento fonosintattico della prima consonante dopo l'articolo o il pronome (cfr.: Loporcaro 1997: 107; Maturi 1997: 12; Rohlfs 1949: 109).

Tornando alla polimorfia fonetica, si osserva un'analoga oscillazione timbrica nella vocale posteriore della preposizione monosillabica «con» (che appare sia come [ko] sia come $[\mathrm{ku}])$, così come nei due bisillabi funzionali ${ }^{1}$ [kom:ə] «come»e $\mathrm{a}$ a'd:o] / [a'ro] «dove, chez» (che presentano le rispettive varianti deaccentate [kum:ə] e [ad:u] / [aru]). ${ }^{2}$

Per quanto riguarda le vocali anteriori, le parole funzionali monosillabiche interessate da un'alternanza tra il grado di apertura medio e quello alto (cioè tra [e] e [i]) sono l'articolo determinativo plurale maschile (7) e femminile (8), i rispettivi pronomi clitici di complemento diretto $(9,10)$ e l'equivalente della preposizione «di» $(11)$.
(7) $[\mathrm{e}$ 'fiK:?]
(8) [e 'fiik:a]
(9) [e 'b:e:kə]
var. [i 'fiא:ə]
var. [i 'fiiא:ə]
var. [i 'b:e:kə]
var. [i 'b:e:kə]
var. [sim: i 'k:a]
«i figli»
«le figlie»
«li vedo»
(10) [e 'b:e:k:]
(11) [sim: e 'k:a]
«le vedo»
«siamo di qua»

Anche in questo caso, come per il singolare maschile e neutro, articoli e pronomi clitici sono omofoni e possiedono un ulteriore allomorfo consonantico qualora la parola successiva inizi per vocale. Negli articoli, la distinzione del genere è di nuovo affidata al rafforzamento fonosintattico (cfr. Loporcaro 1997: 107; Maturi 1997: 12), mentre nei pronomi questa opposizione viene neutralizzata in molte località campane, tra cui Sant'Agata de' Goti (da dove vengono le forme citate) e la stessa Napoli.

1 A rigore, la variazione riscontrata nei due bisillabi non rientra strettamente nella casistica qui esaminata, essendo le vocali in questione lessicalmente accentate; si tratta comunque di una prominenza ridotta a livello di prosodia frasale. Da un punto di vista formale, Bafile (1997b: 462) propone che queste due parole siano «specificate lessicalmente come deaccentabili».

2 A volte i linguisti stessi riproducono involontariamente tale polimorfia, come testimonia l'aneddoto del titolo Comme v'eggia dice? (Maturi 1997) che viene citato in bibliografia da Radtke (1997: 142) nella variante Cumm' eggia dice. 
La grammaticografia descrittiva e normativa, che si ispira spesso a un astratto modello napoletano, predilige gli allomorfi di timbro medio [o] e [e] per articoli determinativi, pronomi e le preposizioni «di» e «come» (v. per esempio Bichelli 1974: 67-68, 128-129 e passim), mentre per l'articolo indeterminativo, la negazione e la preposizione «con» prevale la variante con $[\mathrm{u}]$. In genere tali forme vengono sorrette anche dalla tradizione della scripta; citiamo a caso da alcuni testi della canzone napoletana: 'O paese d' 'o sole, 'E spingole frangese, Voglia 'e turnà, niro comm' a ché, Tu nun 'o saie, Nu iuorno, ecc. Tuttavia non è raro che nella coscienza dei parlanti odierni emergano anche le forme non standard, come ad esempio in un indirizzo di posta elettronica <unapulitane@ecc.>.

\subsection{La polimorfia nei dati dell'AIS}

Un rapido sguardo ad alcune carte dell'Atlante Italo-Svizzero permette di verificare l'oscillazione tra più varianti delle parole funzionali summenzionate. ${ }^{3} \mathrm{~A}$ un primo impatto la polimorfia sembra presentare chiari lineamenti diatopici; ad esempio, la carta 163 «il piede» indica per l'articolo determinativo una sostanziale bipartizione tra dialetti con [o] (per esempio quelli di Napoli e di Ottaviano) e dialetti con [u] (per esempio quelli di Monte di Procida e Formicola) o [lu].

In base ad uno spoglio sistematico dell'AIS - condotto confrontando le realizzazioni degli stessi morfi nelle diverse carte - abbiamo invece scoperto che l'oscillazione delle vocali atone in parole funzionali è presente in ben nove dei quattordici punti di inchiesta, e che l'alternanza tra due forme riguarda ben sette delle undici categorie morfo-lessicali evidenziate in 2.1. (cfr. Maturi / Schmid 2001: 252-253). La variazione non è quindi prevalentemente areale, ma piuttosto inerente ai singoli dialetti locali. Giacché in ogni punto di inchiesta è stato intervistato un solo informatore, questa alternanza rispecchia anche una polimorfia intraindividuale. L'AIS presenta un caso dove i due allomorfi occorrono persino all'interno di uno stesso enunciato: si tratta della carta 1100 («te lo do, se lo vuoi»), dove per la città di Napoli si annota t'u ddongo, si o' vvuo'.

\subsection{Polimorfia in uno stesso parlante e nella stessa località}

L'oscillazione tra due varianti di una stessa parola funzionale è certamente un fenomeno diffuso in buona parte della Campania, per cui l'esempio napoletano dell'AIS può essere considerato la regola piuttosto che l'eccezione. A confermare questa impressione sta la trascrizione di un'intervista con un'informatore di Sant'Agata de' Goti (BN), in cui le vocali medie alternano sistematicamente con quelle alte nelle categorie morfo-lessicali (14) e (7-10), cioè negli articoli e nei pronomi clitici (v. Maturi / Schmid 2001: 254).

3 L'area dei dialetti campani è rappresentata nell'Atlante da 14 punti di inchiesta (i primi due dei quali appartengono oggi alla provincia di Frosinone e quindi alla regione Lazio): 701 San Donato FR, 710 Ausonia FR, 712 Gallo CE, 713 Formicola CE, 714 Colle Sannita BN, 720 Monte di Procida NA, 721 Napoli NA, 722 Ottaviano NA, 723 Montefusco NA, 724 Acerno SA, 725 Trevico AV, 731 Teggiano SA, 740 Omignano SA. 
Per avere un'idea più precisa del rapporto tra forma prevalente e forma minoritaria (Maturi / Schmid 2001: 252) si impone una verifica in termini quantitativi di uno o più campioni dialettali. Un primo passo in questa direzione è consistito nello spoglio delle trascrizioni di una serie di interviste realizzate in cinque località della provincia di Benevento (cfr. 3.1.1.). Nella tabella 1 vengono confrontate, per ognuna delle cinque località, le percentuali delle vocali medie con quelle alte, e ciò nelle due serie delle anteriori $(\mathrm{N}=301)$ e delle posteriori $(\mathrm{N}=420)$.

\begin{tabular}{l|cc|cc}
\hline & $\mathrm{e}$ & $\mathrm{i}$ & $\mathrm{o}$ & $\mathrm{u}$ \\
\hline Amorosi & $20 \%$ & $80 \%$ & $30 \%$ & $70 \%$ \\
Apollosa & $37 \%$ & $63 \%$ & $53 \%$ & $47 \%$ \\
Montefalcone di Val Fortore & $21 \%$ & $79 \%$ & $14 \%$ & $86 \%$ \\
Sant'Agata de' Goti & $41 \%$ & $59 \%$ & $39 \%$ & $61 \%$ \\
Sant'Angelo a Cupolo & $15 \%$ & $85 \%$ & $65 \%$ & $35 \%$ \\
\hline
\end{tabular}

Tab. 1 Articoli e pronomi clitici nel Sannio beneventano: variazione tra vocali medie e alte

La proporzione di [e], [o] rispetto a [i], [u] evidenzia un certo parallelismo nella variazione di altezza tra le due serie di vocali anteriori e posteriori; un'eccezione netta si trova soltanto nell'ultima delle cinque località, Sant'Angelo a Cupolo (cfr. Maturi / Schmid 2001: 254255).

Due osservazioni si impongono a questo punto. Da un lato va sottolineato che i dati riportati sinora sono il risultato dell'interpretazione fatta dagli esploratori, cioè di un'analisi uditiva basata sulla griglia percettiva dei due gradi di apertura alto e medio. Un'analisi acustica, di tipo strumentale, potrebbe invece portare alla luce una distribuzione più articolata di questi morfi nello spazio vocalico, fornendo delle informazioni supplementari sulla loro natura fisica (per esempio sull'intensità, sulla durata ecc.), da mettere eventualmente in relazione con il timbro delle vocali. L'evidente parallelismo nella variazione delle due serie di vocali atone ci porta infatti a cercare una motivazione nelle proprietà fonetico-prosodiche delle singole repliche vocaliche. Cercheremo di approfondire questa ipotesi nei prossimi paragrafi.

\section{L'analisi strumentale della polimorfia}

\subsection{Dati, metodo e risultati preliminari}

3.1.1. Il presente contributo rappresenta la terza fase di una ricerca che durante il suo svolgimento ha visto da un lato un'estensione progressiva della quantità dei dati, dall'altro una continua messa a punto delle ipotesi di analisi.

Nelle prime due tappe abbiamo utilizzato le registrazioni di tre interviste raccolte a Sant'Agata de' Goti (BN) all'interno di un progetto più ampio su dialetto e substandard nel Sannio beneventano (Maturi 1997; 1999; 2000; in stampa a e b). Queste registrazioni fanno parte di un corpus di 36 interviste realizzate in sei località della provincia di Benevento (v. Maturi 1997: 5-6; 1999: 84-86); si tratta di conversazioni semiguidate secondo una metodologia largamente diffusa nelle inchieste sociolinguistiche, vertenti ad esempio su temi della storia locale (la guerra, i terremoti, ecc.), in modo da elicitare un parlato il più 
spontaneo possibile. La varietà adoperata da parte dei tre informatori si colloca verso il polo basilettale del continuum dialetto $\sim$ it. substandard, trattandosi di una coppia di contadini di età superiore ai sessant'anni e di un operaio di circa cinquant'anni che abitano in frazioni periferiche del comune.

3.1.2. Nell'analisi elettroacustica dei dati abbiamo adottato costantemente una identica metodologia. Le misurazioni sono state effettuate al Phonetisches Laboratorium der Universität Zürich, per mezzo di un Sonagraph 5500 della Kay Elemetrics, e riguardano una serie di parametri segmentali e soprasegmentali.

Per determinare il timbro delle vocali abbiamo rilevato i valori delle prime due formanti in Hertz $(\mathrm{Hz}){ }^{4}$ Più precisamente, si è calcolato lo spettro medio dell'intera durata del segmento esaminato; trattandosi di vocali atone e quindi a volte anche molto brevi, abbiamo rinunciato all'idea di isolarne una porzione centrale (che a volte si sarebbe ridotta a uno o due cicli).

Tra i parametri prosodici abbiamo misurato innanzi tutto la durata dei segmenti vocalici in millisecondi $(\mathrm{ms})$ e la loro intensità massima in Decibel $(\mathrm{dB}) ;{ }^{5}$ abbiamo rinunciato a rilevare la frequenza fondamentale (F0), perché non è stato possibile misurarla sistematicamente.

Insieme ai parametri soprasegmentali si è preso inoltre in considerazione il contesto fonotattico di ogni segmento esaminato, annotando il timbro della vocale predecente e di quella successiva.

3.1.3. In una prima fase di verifica strumentale dei dati (v. Maturi / Schmid 1999) abbiamo analizzato 157 repliche appartenenti alle classi morfologiche degli articoli e dei pronomi e distribuite in modo equo sulle due classi fonologiche delle vocali posteriori e anteriori (rispettivamente, 78 e 79 repliche).

Dall'analisi elettroacustica è emersa per entrambe le classi una notevole dispersione soprattutto riguardo all'altezza delle vocali (v. Maturi / Schmid 1999: 1393-1394): i valori della prima formante spaziano da $200 \mathrm{~Hz}$ a $600 \mathrm{~Hz}$ per le posteriori e fino a $640 \mathrm{~Hz}$ per le anteriori, coprendo una gamma che va dalle vocali alte ([u], [i]) alle medio-alte ([o], [e]) fino a comprendere talvolta le medio-basse $([0],[\varepsilon])$. Un primo risultato importante consiste quindi nella constatazione che tanto le vocali posteriori quanto le anteriori si dispongono in un continuum che non presenta nessuna soluzione di continuità tra $\mathrm{i}$ vari gradi di apertura.

Per quanto riguarda invece gli eventuali condizionamenti fonotattici della variazione riscontrata, non si sono potuti riscontrare degli effetti di armonia vocalica veri e propri (cfr. Maturi / Schmid 1999: 1394): in particolare è da notare l'assenza di qualsiasi tipo di assimilazione anticipatoria (o regressiva). Un lieve effetto di coarticolazione sembra realizzarsi piuttosto nella direzione opposta, nel senso che la vocale precedente influisce, seppure non in misura rilevante, sul timbro dei morfi in questione. Tuttavia gli effetti di tale

4 Com'è noto, la prima formante $(\mathrm{F} 1)$ rappresenta il correlato acustico del grado di apertura della vocale, laddove i valori della seconda formante (F2) rispecchiano la posizione della lingua sul piano orizzontale; in altre parole, i valori di F2 sono proporzionali al grado di anteriorità della vocale.

5 Precisiamo che per l'intensità il Sonagraph fornisce dei valori negativi definiti a partire da un livello di energia massima fissato arbitrariamente. 
assimilazione permansiva (o progressiva) incidono prevalentemente sui valori della seconda formante: in linea di massima, una vocale precedente bassa tende a centralizzare il timbro della vocale atona sotto esame.

Se consideriamo le caratteristiche propriamente prosodiche, sorprende che nemmeno la durata delle vocali atone eserciti un effetto chiaro sul loro timbro, almeno non sull'altezza della vocale (cfr. Maturi / Schmid 1999: 1394). Una correlazione - seppure scarsamente significativa - si ottiene semmai con la seconda formante (che non costituisce l'interesse principale della nostra ricerca), ma soltanto nella sottocategoria delle vocali posteriori, dove avviene in effetti una certa riduzione vocalica, nel senso che le realizzazioni più brevi tendono a essere anche più centralizzate.

Più evidenti apparivano invece i risultati per il secondo parametro prosodico, l'intensità, che mostrava delle correlazioni significative con la prima formante per il campione intero e anche per le vocali anteriori e posteriori considerate separatamente (cfr. Maturi / Schmid 1999: 1394). La probabilità che il segmento analizzato abbia un timbro medio (non alto) è dunque maggiore qualora aumenti il volume della voce con cui la vocale in questione viene pronunciata - oppure, detto in altre parole, meno atona è la vocale da un punto di vista fonetico, e più basso è il suo grado di altezza.

3.1.4. Alla luce di questi risultati abbiamo esteso, nella seconda fase della ricerca, la base empirica dei dati (v. Maturi / Schmid 2001). Esaminando sempre la produzione orale degli stessi tre informatori di Sant'Agata de' Goti, abbiamo allargato il corpus a 265 repliche, tra le quali si annoverano non solo occorrenze di articoli determinativi e pronomi clitici, ma anche realizzazioni dei segmenti vocalici delle restanti categorie elencate in 2.1., cioè dell'articolo indeterminativo maschile «un» (5), della negazione «non» (6) e della preposizione «di» (11).

Per questi ultimi tre tipi morfo-lessicali abbiamo riscontrato una gamma di dispersione nello spazio vocalico leggermente diversa (cfr. Maturi / Schmid 2001: 258). Rispetto agli articoli determinativi e ai pronomi clitici di oggetto diretto, qui la variazione della prima formante appare più ristretta: in particolare per l'articolo indeterminativo i valori di F1 vanno da 280 a $580 \mathrm{~Hz}$, per la negazione da 260 a $620 \mathrm{~Hz}$ e per la preposizione «di» da 240 a $480 \mathrm{~Hz}$. D'altro canto, le vocali posteriori mostrano una gamma di dispersione maggiore sul piano della seconda formante, con molti valori attorno ai $1400 \mathrm{~Hz}$. Ciò è un sintomo evidente della parziale centralizzazione della vocale in questi due morfi, la cui struttura fonotattica (CV) contiene anche una consonante. Sembra invece che il processo di centralizzazione venga bloccato quando le parole funzionali consistono di una sola vocale atona.

Per quanto riguarda l'eventuale condizionamento fonetico della variazione timbrica, in questa occasione non è stato preso in considerazione il contesto fonotattico (ovvero la vocale precedente e successiva), mentre abbiamo di nuovo esaminato la durata e l'intensità. Anche in questo campione le durate non mostrano una correlazione significativa con il grado di apertura (v. Maturi / Schmid 2001: 261); tuttavia la gamma di variazione della prima formante si restringe nelle repliche con una durata superiore a $100 \mathrm{~ms}$, addensandosi intorno al valore di $400 \mathrm{~Hz}$ (che corrisponde al timbro di [o]). Comunque la correlazione più significativa va ricercata, anche per il corpus allargato di Sant'Agata de' Goti, nel rapporto tra grado di apertura e intensità, che si manifesta per tutte e cinque le categorie morfosintattiche esaminate in una retta di regressione lineare, il cui coefficiente di $x$ va da 
3,6 nel caso dei pronomi fino a 4,8 per la preposizione «di» (v. Maturi / Schmid 2001: 259260).

In base a questi risultati possiamo quindi affermare che la polimorfia intraindividuale da noi evidenziata a partire dalle descrizioni dialettologiche (cfr. 2.) viene corroborata dall'analisi elettroacustica, dalla quale scaturisce con chiarezza una notevole gamma di variazione timbrica, sotto forma di un continuum lungo l'asse dell'altezza vocalica che spazia dal grado alto a quello medio-basso. Inoltre siamo portati a sostenere che a determinare l'effettivo timbro delle vocali siano in buona misura le caratteristiche prosodiche legate all'esecuzione, di modo che si può parlare di un tipo di polimorfia morfofonetica (v. Maturi / Schmid 2001: 262-263).

3.1.5. Nell'attuale fase della ricerca stiamo procedendo ad una verifica di queste ipotesi sul piano diatopico. Si tratta quindi di esaminare se la polimorfia delle vocali atone nelle parole funzionali costituisca un fenomeno limitato all'area beneventana, o se essa sia invece come pare più probabile in base ai dati AIS (cfr. 2.2.) - diffusa in buona parte della Campania. Inoltre si vuole verificare l'ipotesi di un condizionamento prosodico-fonetico della polimorfia con dati provenienti da altre province.

A tale scopo abbiamo dunque esteso l'area geografica di osservazione, considerando oltre a Sant'Agata de' Goti - anche due località della provincia di Caserta (Bellona e Sparanise) e due della provincia di Napoli (Mugnano e Acerra).

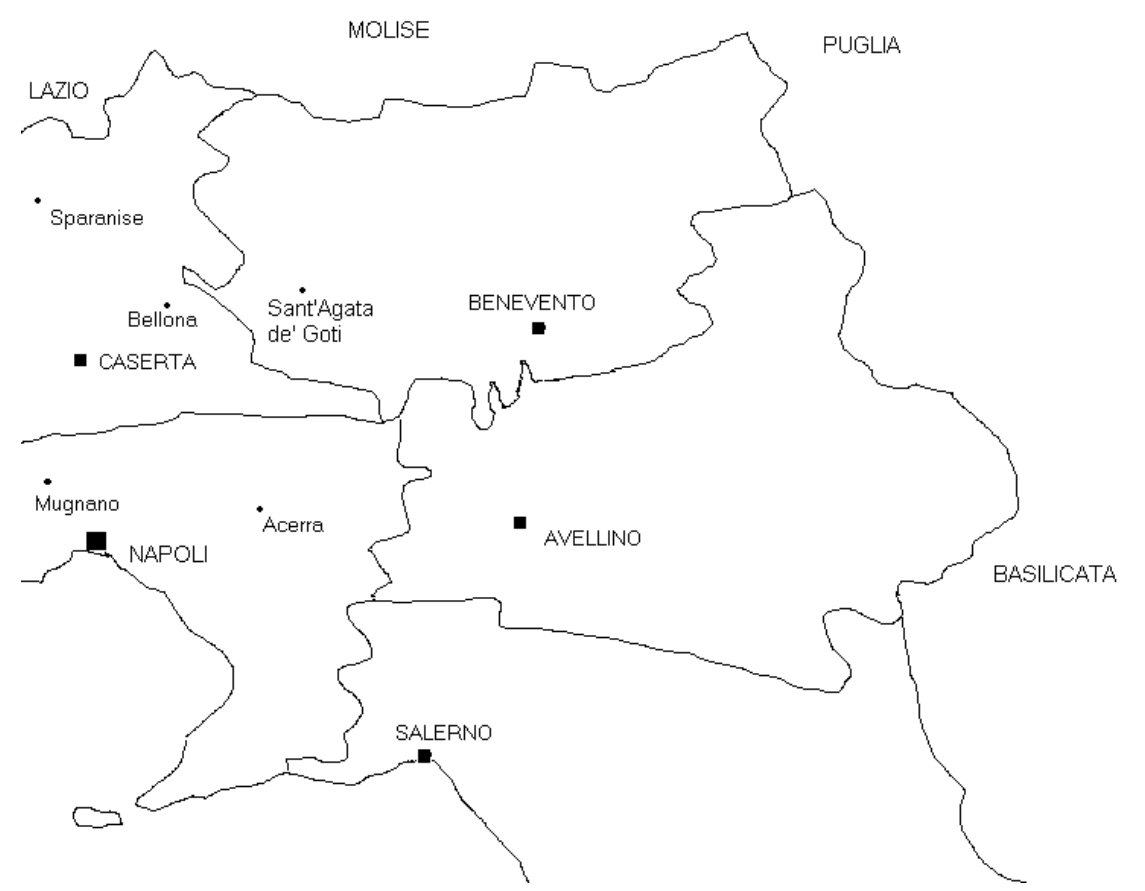

Fig. 2 Posizione geografica dei cinque punti di osservazione della presente indagine

Questo allargamento geografico del corpus non è ancora in grado di coprire l'intera area per la quale si suppone l'esistenza della polimorfia vocalica nelle parole funzionali; in 
particolare rimangono fuori dal raggio di osservazione le due province di Avellino e di Salerno. Comunque possiamo dire che è rappresentata una buona parte del territorio in cui è attestata l'alternanza dei due allomorfi $o$ e $u$ per l'articolo determinativo maschile singolare davanti a consonante (cfr. la carta riportata in Radtke 1997: 82).

Contemporaneamente all'estensione geografica del corpus abbiamo invece ridotto il focus dell'analisi, esaminando soltanto le parole funzionali consistenti di una vocale posteriore (quindi articoli e pronomi al singolare). Questi morfi possono essere considerati prototipici per la polimorfia in questione, in quanto portano il vantaggio di escludere un'eventuale influsso dell'italiano sulla scelta della forma dialettale, che potrebbe invece agire nel plurale maschile di articoli determinativi e pronomi clitici. Siccome in questa sede ci interessa soprattutto la variazione del grado di apertura, sono state escluse anche le parole funzionali che iniziano con una consonante, data la maggiore tendenza alla centralizzazione che appare nelle rispettive vocali (cfr. 3.1.4.)

Per quanto riguarda il tipo di materiale linguistico, abbiamo potuto utilizzare - anche per le località situate nelle province di Caserta e di Napoli - delle registrazioni di parlato spontaneo, prodotto da otto parlanti dialettofoni nativi delle rispettive aree e appartenenti alla fascia d'età tra i 55 e 75 anni. Nel caso della provincia di Caserta si tratta di una coppia di anziani di Bellona e di tre informatori (una donna e due uomini) di Sparanise; per la provincia di Napoli abbiamo esaminato una parlante di Mugnano e due informatori di sesso maschile della località di Acerra. I dati sono stati elicitati a fini non linguistici, in quanto provengono in gran parte da interviste semiguidate, raccolte per il progetto di ricerca «Guerra ai civili. Per un atlante delle stragi naziste in Italia», finanziato dal MURST e dal Dipartimento di Sociologia dell'Università degli Studi di Napoli «Federico II» ${ }^{6}$

Il corpus consiste complessivamente di 172 repliche di vocali posteriori, appartenenti ai primi quattro tipi morfo-lessicali omofoni (articoli determinativi e pronomi clitici maschili singolari e neutri). Le repliche si distribuiscono in modo alquanto disuguale sulle tre province a causa dell'imprevedibilità del numero delle occorrenze nelle diverse interviste: sono più numerosi i dati provenienti dal Sannio beneventano (78 repliche) rispetto a quelli delle province di Caserta e di Napoli (53 e 41 repliche, rispettivamente). Tuttavia, la quantità dei materiali si è rivelata sufficiente per fornire risultati indicativi.

\subsection{La diffusione areale e il continuum acustico della polimorfia vocalica}

L'estensione della base dei dati ci permette di confermare quanto avevamo constatato nelle fasi precedenti. Nell'articolo determinativo singolare maschile e neutro, così come nei pronomi clitici omofoni, l'apertura della vocale varia dal grado alto $[\mathrm{u}]$ a quello mediobasso [0], lungo un continuum centrato attorno al grado medio-alto [o]. Risulta quindi che tale dispersione dei morfi nello spazio vocalico non è affatto limitata al Sannio beneventano, ma appare in maniera pressoché identica in un'area geografica molto più vasta.

6 Ringraziamo la Prof. Gabriella Gribaudi per aver concesso l'uso dei materiali. A Bellona, due interviste sullo stesso argomento sono state realizzate indipendentemente da Davide Piscitello dell’Università di Zurigo, che qui ringraziamo ugualmente. 


\begin{tabular}{lllll}
\hline & Benevento & Caserta & Napoli & Corpus intero \\
\hline min, max & 200,600 & 240,560 & 260,560 & 200,600 \\
media (dev. standard) & $406( \pm 76)$ & $409( \pm 80)$ & $402( \pm 61)$ & $407( \pm 78)$ \\
\hline
\end{tabular}

Tab. 3 Valori di F1 nelle vocali atone (in Hz). Confronto tra le tre province e il corpus intero

Come si evince dalla tabella 3, un confronto fra le tre province e il corpus intero rivela per Sant'Agata de' Goti dei valori minimi e massimi leggermente più divaricati, il che è dovuto con molta probabilità al maggior numero delle repliche. Infatti, se consideriamo la media e la deviazione standard, il sottoinsieme beneventano risulta essere altamente rappresentativo di tutto il campione campano.

La dispersione dei valori formantici è in buona misura coestensiva nei dati delle tre province, come emerge in modo chiaro dalla figura 4. Tralasciando in questa sede le differenze sul piano di F2 (dove le repliche casertane mostrerebbero una tendenza lievemente maggiore alla centralizzazione), teniamo invece a ribadire il forte aspetto di continuum che si manifesta nella rappresentazione di questi dati: le repliche sono distribuite in maniera abbastanza regolare su tutta l'area di dispersione, con un certo addensamento soltanto intorno ai valori medi di $407 \mathrm{~Hz}$ (F1) e di $868 \mathrm{~Hz}$ (F2). Notiamo, a titolo di curiosità, che un confronto con i valori forniti da Albano Leoni / Maturi (1995: 96) per l'italiano mostra una coincidenza dei nostri dati con le medie della prima formante per [o] tonica $(409 \mathrm{~Hz})$ e della seconda formante per $[\mathrm{u}]$ tonica $(861 \mathrm{~Hz})$.

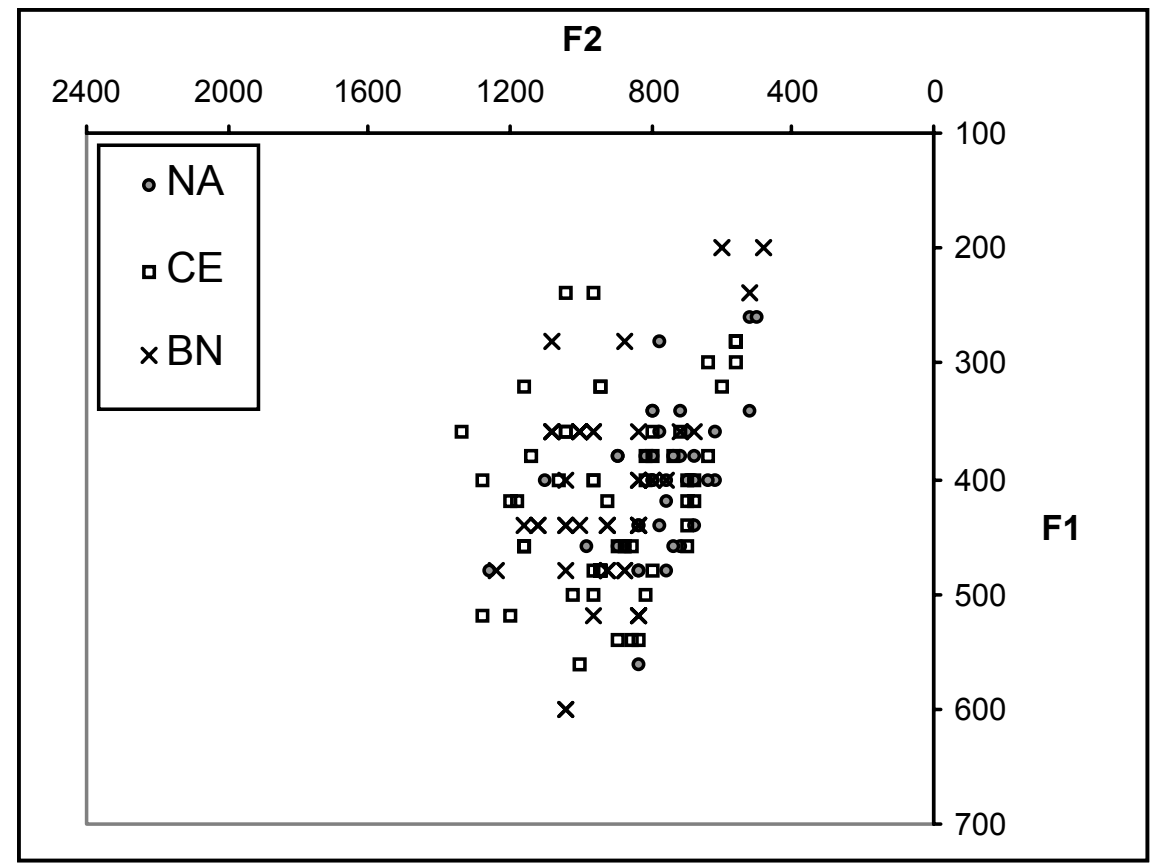

Fig. 4 Dispersione di articoli e pronomi nello spazio vocalico. Confronto fra le tre province 


\subsection{Il condizionamento prosodico della polimorfia vocalica}

3.3.1. Data la scarsa rilevanza del contesto fonotattico emersa nella prima fase della ricerca (cfr. 3.1.3.), abbiamo trascurato per la presente analisi il timbro delle vocali precedenti e successive.

3.3.2. Abbiamo invece tenuto conto, anche in questa sede, del parametro durata.

\begin{tabular}{lllll}
\hline & Benevento & Caserta & Napoli & Corpus intero \\
\hline min, $\max$ & 31,305 & 36,514 & 23,314 & 23,514 \\
media (dev. standard) & $80( \pm 45)$ & $95( \pm 77)$ & $92( \pm 73)$ & $85( \pm 68)$ \\
\hline
\end{tabular}

Tab. 5 Durata delle vocali atone (in $\mathrm{ms}$ ). Confronto fra le tre province e il corpus intero

Come si vede dalla tabella 5 , vi è una notevole differenza tra $\mathrm{i}$ valori minimi e quelli massimi, dovuta soprattutto alla presenza di alcune repliche con una durata molto lunga; evidentemente, in questi casi l'allungamento della vocale viene prodotto da un momento di esitazione del parlante nella pianificazione del suo enunciato. Si riscontra quindi una forte variabilità delle durate in tutte e tre le province, evidente anche nel rapporto relativamente alto tra la deviazione standard e le rispettive medie. Comunque i tre sottocampioni non si differenziano sensibilmente tra di loro, nonostante la media e la deviazione standard un po' più basse nei dati beneventani. Notiamo a questo punto che i valori sono comunque relativamente alti per delle vocali atone e si posizionano in qualche modo in una zona intermedia tra le durate misurate per le vocali atone e quelle per le toniche dell'italiano - in sillaba aperta, 40-70 ms e 90-150 ms, rispettivamente (cfr. Marotta 1985: 160-169).

Il diagramma 6 rappresenta la durata in funzione grado di apertura.

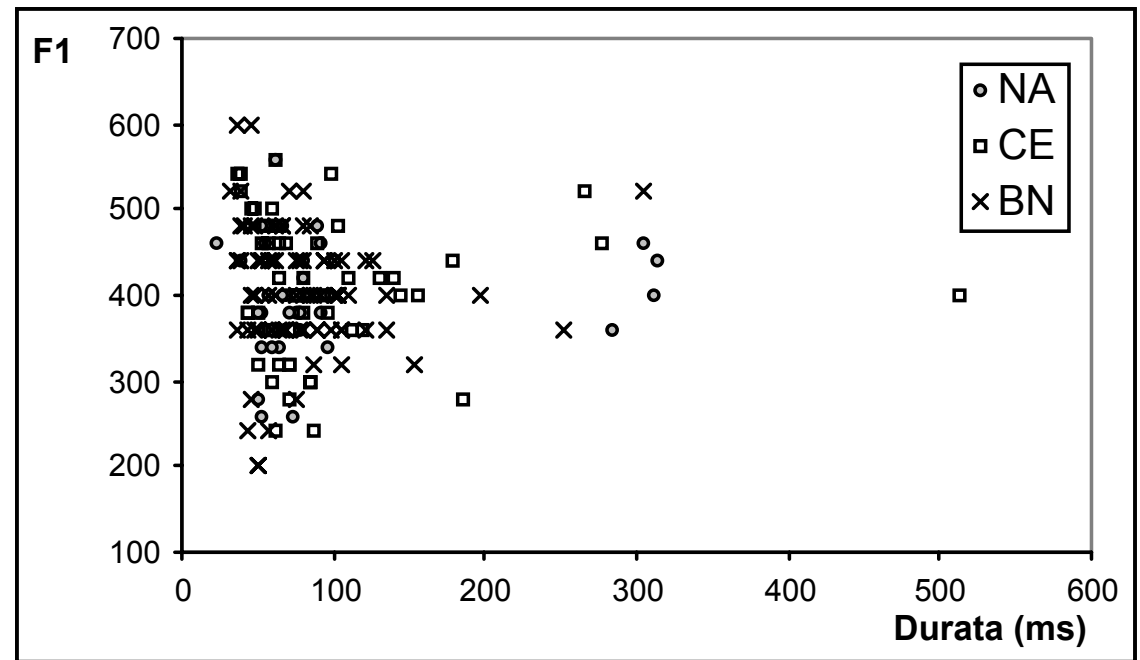

Fig. 6 Durate e F1. Confronto fra le tre province

Anche qui la distribuzione delle occorrenze porta a escludere l'esistenza di una correlazione diretta tra durata e F1 (cfr. 3.1.4.). Ciononostante, dalla figura 6 emerge - in modo ancora più chiaro che non nella fase precedente della nostra ricerca (cfr. Maturi / Schmid 2001: 
261) - una distribuzione delle vocali di tipo triangolare, nella quale la gamma di variazione di F1 si restringe man mano che le durate aumentano. In altre parole, più lunga è la vocale e più essa si avvicina - tendenzialmente - a quello che potremmo considerare il suo target ovvero la forma profonda, cioè $/ \mathrm{o} /$.

3.3.3. Passiamo infine a quella dimensione prosodica che nelle fasi precedenti della ricerca ha fornito le correlazioni più significative con la variazione di altezza delle vocali: l'intensità.

\begin{tabular}{lllll}
\hline & Benevento & Caserta & Napoli & Corpus intero \\
\hline min, max & $-44,-7$ & $-37,-13$ & $-33,-20$ & $-44,-7$ \\
media (dev. standard) & $-27( \pm 8)$ & $-28( \pm 5)$ & $-28( \pm 5)$ & $-27( \pm 7)$ \\
\hline
\end{tabular}

Tab. 7 L'intensità delle vocali atone (in $\mathrm{dB}$ ). Confronto fra le tre province e il corpus intero

Come si evince dalla tabella 7 , i dati beneventani presentano una dispersione dei valori in $\mathrm{dB}$ leggermente maggiore (il che sarà anche in questo caso da ricondurre alla quantità maggiore delle repliche), ma mostrano di essere altamente rappresentativi se consideriamo le medie (e le rispettive deviazioni standard) delle restanti due province e del corpus intero.

Meno uniforme è il quadro fornito dal grafico 8 , nel quale la dimensione dell'intensità viene proiettata contro il grado di apertura.

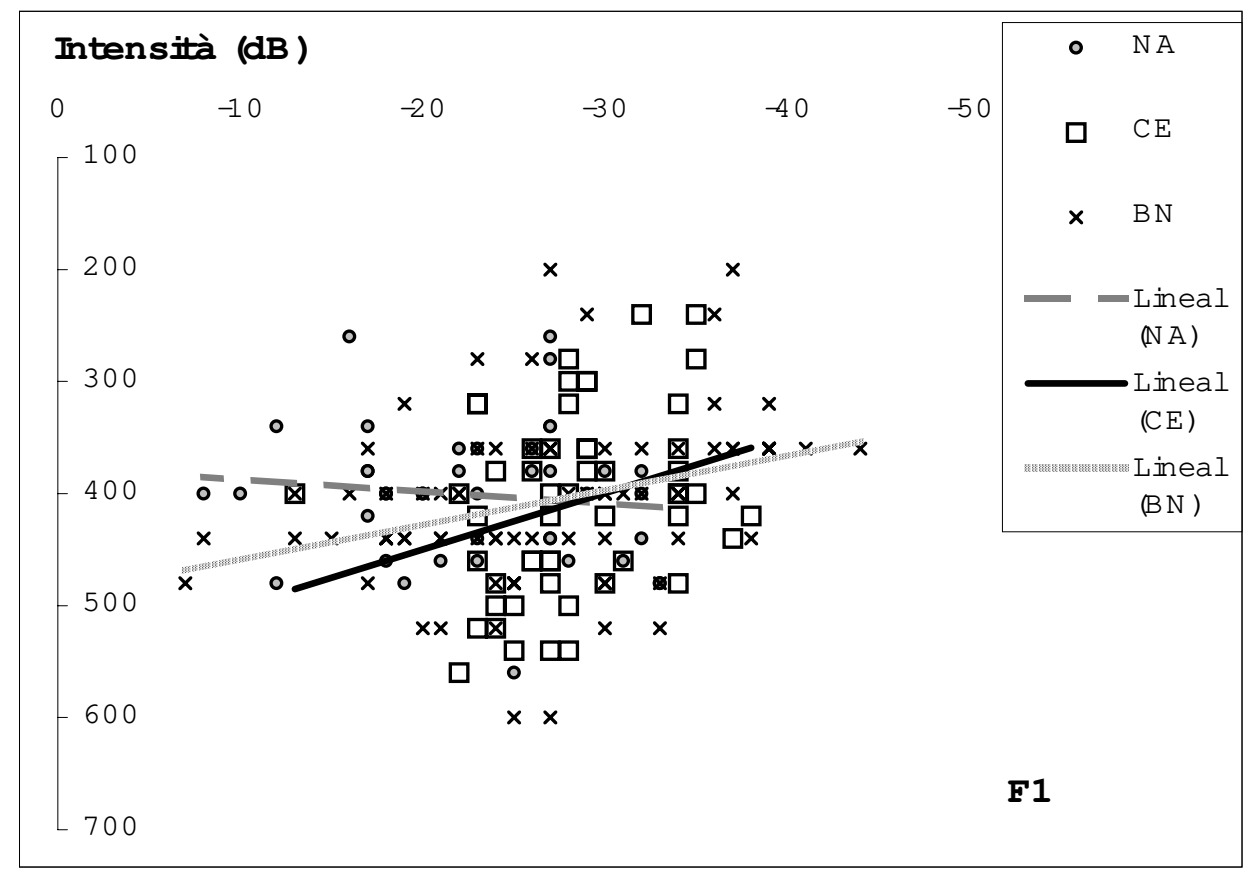

Fig. 8 Correlazione tra intensità e F1. Confronto fra le tre province

Secondo la nostra ipotesi, nelle parole funzionali le vocali tendono ad essere meno alte quando esse sono pronunciate con un volume della voce maggiore. Ora, questa tendenza sembra sì realizzarsi nel corpus considerato nella sua totalità, ma essa non si manifesta in 
maniera uguale nei dati delle tre province prese in considerazione. Se è vero che il sottocampione casertano dà luogo a una retta di regressione ancora più ripida di quella degli stessi dati beneventani, nei dati della provincia di Napoli la correlazione sembrerebbe invece essere di segno opposto (benché in misura assai poco significativa).

\section{Osservazioni conclusive}

Tirando le somme, ci sembra comunque di poter affermare che l'obiettivo principale di questa terza fase della nostra ricerca è stato ampiamente raggiunto. In particolare viene confermata l'ipotesi riguardo alla diffusione areale della variazione morfo-fonetica esaminata: è ormai evidente che la polimorfia delle parole funzionali non è un fenomeno ristretto al Sannio beneventano, ma che l'oscillazione nell'altezza delle vocali atone si presenta in proporzioni e modalità analoghe in buona parte dei dialetti campani (almeno nelle province di Napoli, Caserta e Benevento). Dal punto di vista della loro caratterizzazione fonetica, queste vocali formano un continuum che si estende ininterrottamente nello spazio vocalico a partire dal grado di apertura alto fino a raggiungere quello medio-basso.

In linea di massima viene confermata anche l'interdipendenza tra il contenuto segmentale e le proprietà prosodiche delle vocali atone: la gamma di variazione si restringe con l'aumentare delle durate e tendenzialmente si delinea un rapporto inversamente proporzionale tra intensità e altezza vocalica (eccezion fatta per i dati provenienti dalla provincia di Napoli).

In altre sedi abbiamo sottolineato l'interesse teorico suscitato dall'idea di un'interfaccia diretta tra fonetica e morfologia (cfr. Maturi / Schmid 1999: 1395; 2001: 263). In questa prospettiva, la dimensione fonetico-prosodica - osservabile nella produzione linguistica concreta dei parlanti attraverso i parametri della durata e dell'intensità - si troverebbe ad interagire con delle restrizioni di natura morfologica che ammettono una certa variazione di altezza soltanto nelle vocali di un numero limitato di parole funzionali.

È però altrettanto evidente che la polimorfia di queste vocali scaturisce da una tensione inerente al sistema fonologico dei dialetti campani, in cui la forma soggiacente della rappresentazione lessicale /o/ viene a scontrarsi con un processo generale di innalzamento delle vocali medio-alte protoniche (cfr. Bafile 1997a: 145, n. 22; Maturi / Schmid in stampa). Questo spontaneo passaggio a $[\mathrm{u}]$ viene quindi ostacolato - sia pure in modo parziale e variabile - dalla prominenza prosodica (in termini di durata e intensità) che a queste vocali atone può atipicamente essere assegnata, a causa della rilevanza comunicativa delle parole funzionali che esse rappresentano. 


\section{Bibliografia}

AIS (1928-1940): = Karl Jaberg, Jakob Jud, Sprach- und Sachatlas Italiens und der Südschweiz. Zofingen: Ringier.

Albano Leoni, Federico, Pietro Maturi (1995): Manuale di fonetica. Roma: La Nuova Italia Scientifica.

Bafile, Laura (1997a): L'innalzamento vocalico in napoletano: un caso di interazione tra fonologia e morfologia. In: Luciano Agostiniani et al. (a cura di): Atti del III Convegno della Società Internazionale di Linguistica e Filologia Italiana. Napoli: Edizioni Scientifiche Italiane, 127-148.

- (1997b): Parole grammaticali e struttura prosodica: dati dell'italiano e del napoletano. In: LeSt 32, 433-469.

Bichelli, Pirro (1974): Grammatica del napoletano. Bari: Pégaso.

Loporcaro, Michele (1997): L'origine del raddoppiamento fonosintattico. Saggio di fonologia diacronica romanza. Basel, Tübingen: Francke Verlag.

Marotta, Giovanna (1985): Modelli e misure ritmiche: la durata vocalica in italiano. Bologna: Zanichelli.

Maturi, Pietro (1997): Comme v'eggia dice. Testi orali dal Sannio beneventano in trascrizione fonetica. Kiel: Westensee.

- (1999): Dialetti e substandard. Linea guida di una ricerca empirica nel Sannio beneventano. In: Gianna Marcato (a cura di): Dialetti oggi. Padova: Unipress, 83-91.

- (2000): Per un modello dinamico del continuum dialetto lingua: dati dal Sannio beneventano. In: ACILPR XXII, vol. 3, 247-254.

- (in stampa a): Dialetti e substandardizzazione nel Sannio beneventano. Frankfurt: Peter Lang.

- (in stampa b): Variabilità morfofonetica nei dialetti del Sannio beneventano. In: Atti del V Convegno della Società Internazionale di Linguistica e Filologia Italiana.

Maturi, Pietro, Stephan Schmid (1999): Phonetically conditioned allomorphy of functional words in a dialect of Southern Italy. In: Proceedings of the $14^{\text {th }}$ International Congress of Phonetic Sciences. Berkeley: University of California, 1393-1396.

- (2001): Allomorfia e morfo-fonetica: riflessioni induttive su dati dialettali campani. In: Federico Albano Leoni et al. (a cura di): Dati empirici e teorie linguistiche. Roma: Bulzoni, 251-265.

- (in stampa): Dialettologia e fonetica acustica. Una ricerca in Campania. In: Agostino Regnicoli (a cura di): La fonetica acustica come strumento di analisi della variazione linguistica in Italia. Atti delle XII Giornate di Studio del Gruppo di Fonetica Sperimentale. Roma: Esagrafica.

Radtke, Edgar (1997): I dialetti della Campania. Roma: Il Calamo.

Rohlfs, Gerhard (1968): Grammatica storica della lingua italiana e dei suoi dialetti. II. Morfologia. Torino: Einaudi. (1949) 